\title{
Los corticoides inhalatorios reducirian la mortalidad de los pacientes con enfermedad pulmonar obstructiva crónica
}

Sin y col. Thorax. 2005 Dec;60(12):992-7.

\section{Objetivo}

Explorar el impacto a largo plazo de los corticoides inhalatorios (Cl) sobre la mortalidad general de los pacientes con enfermedad pulmonar obstructiva crónica (EPOC).

\section{Fuente y selección de datos}

Análisis por intención de tratar de datos de siete estudios publicados con más de 12 meses de seguimiento, que compararon el uso de $\mathrm{Cl}$ contra placebo en pacientes con EPOC estable.

\section{Resultados principales}

Se analizaron los datos de 5085 pacientes con una media de edad de 59 años $(\mathrm{DE} 9,3)$ y una media de seguimiento de 26 meses (DE15). El $71 \%$ de la muestra eran hombres y el $69 \%$, fumadores. Al ingreso, el $9 \%$ de los pacientes eran EPOC estadio IV, $28 \%$ estadio III, $48 \%$ estadio II y $15 \%$ estadio I. A lo largo del estudio la mortalidad global fue $4 \%$ (n:201).

Los hombres tuvieron una mortalidad estadísticamente superior a las mujeres $(4,5 \%$ vs. $2,6 \% p=0,002)$.

Los pacientes que recibieron $\mathrm{Cl}$ tuvieron una mortalidad menor que que los asignados a placebo (HR 0,75; IC95\% 0,57-0,99). El ajuste por sexo, edad, tabaquismo activo, índice de masa corporar y volumen espiratorio forzado en el primer segundo (VEF1) basal luego de la inhalación de broncodilatador no mostró cambios importantes en los resultados (HR 0,73; IC95\% 0,55-0,96).

En el análisis de subgrupos hubo una tendencia beneficiosa de los $\mathrm{Cl}$ especialmente notable en las mujeres (HR 0,46; IC95\% 0,240,91 ) en los fumadores activos (HR 0,60; IC 95\% 0,39-0,93) y en aquellos pacientes con un VEF1 basal menor luego de la inhalación de broncodilatador menor a $60 \%$ del esperado por edad y antropometría (HR 0,67 IC95\% 0,48-0,94) aunque estos resultados tienen que analizarse con precaución.

El efecto beneficioso de los $\mathrm{Cl}$ fue similar para los distintos tipos utilizados (fluticasona, budesonida y triamcinolona).

\section{Conclusiones}

El uso de corticoides inhalatorios a largo plazo reduce la mortalidad general de los pacientes con EPOC, aunque hacen falta estudios que demuestren la persistencia de estos beneficios después de dos a tres años.

Fuente de financiamiento y posibles conflictos de intereses: Canadian Institutes of Health Research, Ottawa, and Alberta Heritage Foundation for Medical Research, Edmonton, Canada. Los autores reciben o recibieron honorarios de Astra Zeneca y Glaxo Smith Kline en calidad de disertantes esponsoreados por dichas empresas o como empleados de las mismas.

\section{Comentario}

Hasta la fecha de publicación del estudio de Sin DD y col, la cesación tabáquica ${ }^{1}$ y la oxigenoterapia ${ }^{2}$ eran las únicas medidas terapéuticas con eficacia demostrada para el tratamiento de los pacientes con EPOC, en quienes había evidencia a favor de la modificación de su pronóstico de vida. Los estudios que habían intentado encontrar reducción de la mortalidad asociada al uso de corticoides inhalatorios en esta población no tenían el poder suficiente para poder demostrar diferencias ${ }^{3,4,5}$ lo que avala el análisis global de estos trabajos en forma agrupada.

La gran fortaleza del estudio que comentamos es que recolectó y analizó agrupadamente las bases de datos de siete estudios prospectivos aleatorizados y controlados con placebo de más de 12 meses de seguimiento; lo que permitió obtener un número suficiente de pacientes para poder detectar diferencias estadísticamente significativas de $27 \%$ en la tasa de mortalidad (IC95\% 4$45 \%$ ). Meta-análisis previos publicados sobre el uso corticoides inhalatorios en el manejo de la EPOC ${ }^{6}$ habían evidenciado que el uso de $\mathrm{Cl}$ disminuye la tasa de exacerbaciones, pero no habían mostrado diferencias estadísticas en la mortalidad de esta población de pacientes (RR 0,84; IC95\% 0,6-1,18).

De acuerdo a los resultados del estudio que comentamos, para evitar una muerte sería necesario tratar con corticoides inhalatorios durante 26 meses a 189 pacientes con EPOC de estas características. Una debilidad del reporte es que no queda claro el motivo por el cual los autores excluyeron del análisis algunos estudios ${ }^{7}$ que cumplieron los criterios de inclusión. Tampoco aclaran detalladamente cómo hicieron la búsqueda bibliográfica y por que seleccionaron sólo siete estudios para el análisis. Estos interrogantes dejan abierta la duda de cómo controlaron los sesgos de publicación* y/o selección*, que pueden existir en este tipo de investigación.

\section{Conclusión del comentador}

En el mediano plazo, los corticoides inhalatorios reducirían la mortalidad de los pacientes con enfermedad pulmonar obstructiva crónica.

Pablo Jinich [ Médico Especialista en Medicina Familiar. Unidad de Medicina Familiar y Preventiva del Hospital Italiano de Buenos Aires. ]

Jinich P. Los corticoides inhalatorios reducirían la mortalidad de los pacientes con enfermedad pulmonar obstrucutiva crónica. Evid. actual. pract. ambul. 9(3);71. May-Jun. 2006. Comentado de: Sin DD, Wu L, Anderson JA, Anthonisen NR, Buist AS, Burge PS, Calverley PM, Connett JE, Lindmark B, Pauwels RA, Postma DS, Soriano JB, Szafranski W, Vestbo J. Inhaled corticosteroids and mortality in chronic obstructive pulmonary disease. Thorax. 2005 Dec;60(12):9927. PMID: 16227327

\section{Referencias}

1. Anthonisen NR, Skeans MA, Wise RA, Manfreda J, Kanner RE, Connett JE; Lung Health Study Research Group. The effects of a smoking cessation intervention on 5-year mortality: a randomized clinical trial. Ann Intern Med. 2005 Feb 15;142(4):233-9

2. Nocturnal Oxygen Therapy Trial Group.Continuous or nocturnal oxygen therapy in hypoxemic chronic obstructive lung disease: a clinical trial. Ann Intern Med. 1980 Sep;93(3):391-8.

3. Lung Health Study Research Group. Effect of inhaled triamcinolone on the decline in pulmonary function in chronic obstructive pulmonary disease. $\mathrm{N}$ Engl $\mathrm{J}$ Med 2000:343:1902-9.

4. Pauwels RA, Lofdahl CG, Laitinen LA, et al. Long-term treatment with inhaled budesonide in persons with mild chronic obstructive pulmonary disease who continue smoking. European Respiratory Society Study on Chronic Obstructive Pulmonary Disease. N Engl J Med 1999;340:1948-53

5. Burge PS, Calverley PM, Jones PW, et al. Randomised, double blind, placebo controlled study of fluticasone propionate in patients with moderate to severe chronic obstructive pulmonary disease: the ISOLDE trial. BMJ 2000;320:1297-303

6. Alsaeedi A, Sin DD, McAlister FA The effects of inhaled corticosteroids in chronic obstructive pulmonary disease: a systematic review of randomized placebo-controlled trials.Am J Med. 2002 Jul;113(1):59-65.

7. Weir DC, Bale GA, Bright P, Sherwood Burge P. A double-blind placebo-controlled study of the effect of inhaled beclomethasone dipropionate for 2 years in patients with nonasthmatic chronic obstructive pulmonary disease.Clin Exp Allergy. 1999 Jun;29 Suppl 2:125-8. 